\title{
Business Plans
}

\author{
Timothy P. Murphy, M.D., F.S.I.R., F.A.H.A., F.S.V.M.B., ${ }^{1}$ \\ and Gregory M. Soares, M.D.'
}

\begin{abstract}
The business strategy for establishing a clinical office involves three key components: fundamental change from the traditional technical model in processes for accepting referrals; establishment of a high-quality clinical operation; and changing the perception of interventionalist radiology among the referring community and the public. The costs and revenues used to derive profit and loss statements will vary considerably across regions and practice set-ups. Some of the considerations are outlined in this article, but it will be up to interventionalists and their business managers to make these factors conform to their individual practices and locations.
\end{abstract}

KEYWORDS: Strategy, outpatient referral, pro forma, market, risk

Objectives: Upon completion of this article, the reader will gain a basic understanding of the standard business proposal for establishing a new clinical office.

Accreditation: Tufts University School of Medicine (TUSM) is accredited by the Accreditation Council for Continuing Medical Education to provide continuing medical education for physicians.

Credit: TUSM designates this educational activity for a maximum of 1 Category 1 credit toward the AMA Physicians Recognition Award. Each physician should claim only those credits that he/she actually spent in the activity.

In this section we review a standard proposal for establishing a clinical office, focusing on the costs associated with such a venture and how the clinical practice should be structured. This proposal is based on the assumption that interventional radiologists will usually want to have hospital privileges and the ability to use imaging equipment and other resources in a hospital to perform operative procedures. Although clinical practice in the hospital, such as admitting patients and performing rounds on both service and consult patients, are essential components of a clinical practice model, it will not be the focus of this proposal. The business strategy involves three key components: fundamental change from the traditional technical model in processes for accepting referrals; establishment of a high-quality clinical operation; and changing the perception of inter- ventional radiology among the referring community and the public.

Some of the items that must be addressed within a group practice include:

1. Establishment of a clinic rotation to serve the referring population associated with the associated hospital(s), including office space, office staff, and physician rotation to accommodate an outpatient clinical service.

2. Hiring physician extender(s) to assist with the medical office and expanded inpatient services.

3. Understanding that a clinical practice will require patients to be seen by in consultation by an interventional radiologist doctor prior to scheduling therapeutic interventions.

Clinical Practice Development; Editors in Chief, Brian Funaki, M.D., Peter R. Mueller, M.D.; Guest Editors, Timothy P. Murphy, M.D., F.S.I.R., F.A.H.A., F.S.V.M.B., Gregory M. Soares, M.D. Seminars in Interventional Radiology, volume 22, number 1, 2005. Address for correspondence and reprint requests: Timothy P. Murphy, M.D., F.S.I.R., F.A.H.A., F.S.V.M.B., Associate Professor of Diagnostic Imaging, Brown Medical School, Rhode Island Hospital, 593 Eddy Street, Providence, RI 02903. ${ }^{1}$ Brown Medical School, Rhode Island Hospital, Providence, Rhode Island. Copyright (C) 2005 by Thieme Medical Publishers, Inc., 333 Seventh Avenue, New York, NY 10001, USA. Tel: +1(212) 584-4662. 07399529,p;2005,22,01,045,048,ftx,en;sir00288x. 
4. Consideration of logistical issues such as fixed schedules to accommodate patient continuity.

5. Support for start-up office costs.

6. Providing marketing resources for interventional radiology practice promotion efforts in the range of 5 to $10 \%$ of gross revenues.

\section{OFFICE LOCATIONS}

Outpatient referrals are almost always elective. Elective, outpatient consults are usually for disease processes that are often treated with complex invasive procedures, which include patients with peripheral arterial and venous disease, women's reproductive care issues, neurovascular disease, and cancer. These diseases are interesting to treat, offer considerable impact on patient outcomes, and are well reimbursed. This business plan is designed to increase the referrals of these patients. Conversely, if a clinical practice model is not implemented, these referrals are likely to decrease in the future.

A clinical practice cannot be fully conducted in a hospital, least of all in a radiology department. As noted in a previous article in this issue, the federal antikickback statute of 1972 prevents hospitals from providing noncompensated office space to doctors. Therefore, an office separate from the hospital is essential. It will be most convenient to locate the office near the hospital so it is convenient to go back and forth between the two. Some practices may want multiple offices to serve multiple population areas or hospitals. If the office practice must be fragmented, it may be desirable to look for a shared office or to sublet space in an office to reduce overhead. The disadvantage of this is that there may be restrictions on signage and therefore promotion of the office could be limited. Leases should be carefully crafted to avoid the inability to expand or contract the clinical service as needed.

\section{FINANCIALS: AMBULATORY CLINIC/MEDICAL OFFICE}

Many practices would like to know upfront about the return on investment for establishing a clinical interventional radiology practice. It is important to try and establish projections and estimates for this, but it must be emphasized that patient care comes first. There are compelling ethical reasons why interventional radiologists should not perform therapeutic procedures without establishing a meaningful physician-patient relationship, which can only be done within a clinical practice-even if it means that an office itself will lose money.

The pro forma provided (see Table 2) is for a hypothetical private medical office operated by a physician private practice. It includes reasonable but conservative estimates of costs. The pro forma is suitable for a start-up office practice that would fill 1 full clinic day per week. It assumes that an interventional radiologist would be available in the clinic one-half day per week and 1 full day per week in an interventional radiology department performing procedures generated in the clinic. It assigns only $20 \%$ of physician assistant (PA) effort to this hypothetical venture, as the PA would work only 1 day per week in the clinic. This pro forma is conservative for several reasons. It does not consider other revenue generated for the practice by imaging tests ordered on patients seen in the clinic, which may occur in up to $70 \%$ of patients. ${ }^{1}$ And because the pro forma is for a start-up office practice in session only 1 day per week, $80 \%$ of the overhead could be used more efficiently (such as using part-time office help, or subleasing space when the office is not in session). Even with these conservative assumptions, however, the pro forma shows a profit of more than $\$ 100,000$ for an office practice seeing patients 1 day per week with six new patients referred per week. Ventures smaller than this hypothetical one may consider subleasing or sharing office space and staff. Other procedures, such as lower extremity varicose vein treatment, may also be offered in the space, increasing the practice time spent in the office to at least 2 or 3 days per week. As many of the costs are fixed, growth can be accommodated with more proportional contribution to profit.

The costs and revenues used to derive profit and loss statements will vary considerably across regions and practice setups. Some of the considerations are outlined below, but it will be up to interventionalists and their business managers to make these factors conform to their individual practices and locations. Financial assumptions (Table 1) and a pro forma based on them (Tables 2 and 3) are provided.

\section{The Potential Market}

The Department of Health and Human Services projects that there are $\sim 100,000$ peripheral artery stent procedures performed in the United States each year, in addition to 80,000 carotid endarterectomies and 70,000 superficial femoral artery bypass grafts. There are as many as 9 million claudicants in the United States, of which at least $35 \%$ have disease amenable to endovascular revascularization, given pending technologies. This population could amount to 100,000 procedures annually. There are 2 million Americans with renal artery stenosis; if the National Institutes of Health CORAL study is accurate, there could be 200,000 renal stent procedures per year in the United States. Between carotids, renals, iliacs, and femoral arteries, there could be as many as 500,000 procedures performed in the United States each year. Approximately 200,000 women undergo hysterectomies for uterine fibroids each year. There are $\sim 20,000$ cases of primary liver cancer and 
Table 1 Revenue Assumptions*

\begin{tabular}{|c|c|c|}
\hline Service Description & Reimbursement & \\
\hline Fibroid embolization procedure & $\$ 2,011$ & \\
\hline lliac, femoral, or renal artery stent & $\$ 1,154$ & \\
\hline Hepatic artery chemoembolization & $\$ 1,519$ & \\
\hline Low-level follow-up (99212) & $\$ 30$ & \\
\hline Mid-level consult (99243) & $\$ 110$ & \\
\hline Expenditures & \$/Month & $\underline{\$ / Y e a r}$ \\
\hline Rent (1200 sq. ft @ \$22.50/sq.ft. $)^{\dagger}$ & $\$ 2,250$ & $\$ 27,000$ \\
\hline Amortized buildout, furniture, and supplies ${ }^{\ddagger}$ & $\$ 1,742$ & $\$ 20,900$ \\
\hline Medical aid ${ }^{\S}$ & $\$ 2,808$ & $\$ 33,696$ \\
\hline Physician's assistant" & $\$ 1,521$ & $\$ 91,247$ \\
\hline Medical receptionist & $\$ 1,779$ & $\$ 21,344$ \\
\hline Interventional radiologist ${ }^{\#}$ & $\$ 15,513$ & $\$ 347,613$ \\
\hline Computer hardware and information systems ${ }^{*}$ * & $\$ 990$ & $\$ 11,880$ \\
\hline Billing and collections ${ }^{\dagger \dagger}$ & $\$ 1,175$ & $\$ 14,099$ \\
\hline
\end{tabular}

*Based on 8 hours per day, 21 workings days/month.

$\$$ \$15-30/sq. ft./y, used \$22.50; http://www.spaceforlease.com/PropertySearch.asp?Prop_Type $=110$.

Estimated at $\$ 100,000-200,000$ over 10 years at $7 \%$ (used $\$ 150,000$ ).

Medicare fee schedule.

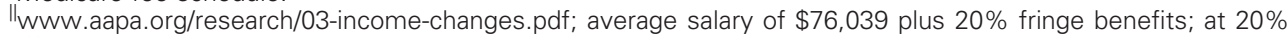
effort.

TMedical.receptionist.jobs.monster.com (\$9-14/hr, used \$11.50).

\#swz.salary.com/salarywizard/layouthtmls/swzl_compresult_national_HC07000062.html; \$261,363, plus 33\% benefits; interventional radiologists' time is assigned at 1.5 days per week to include 0.5 days per week in the clinic and 1 day per week to perform the procedures for 52 weeks; also assuming 1.6 full-time equivalents to accommodate time off.

$* * \$ 10,000-100,000$, used $\$ 50,000$ over 5 years at $7 \%$.

$\dagger \dagger \%$ of revenues.

probably at least an equal number of cases of metastatic liver disease diagnosed each year. Roughly $40 \%$ of middle-aged women have symptomatic lower extremity superficial venous insufficiency requiring some form of treatment. In the future, many of these patients will be treated with endovascular therapy, conservatively estimated to generate 500,000 to 1 million high-end services annually. To put all this into perspective, this is comparable to the volume of coronary artery interventions done in the United States each year. The downside to underexpanding and underpromoting clinical services is potentially enormous.

\section{Staffing Needs}

A medical office will require at least one office person to arrange appointments and help schedule examinations and referrals when the office is open. This can be a parttime employee who works elsewhere when the office is closed. However, because referring physicians may want

Table 2 Pro Forma

\begin{tabular}{llll}
\hline $\begin{array}{l}\text { Projected Average Volume Generated } \\
\text { With 1 Full Day in Clinic Per Week* }\end{array}$ & Monthly & Daily & Monthly \\
\hline Low-level follow-up (99212) & 64.8 & 18 & 81 \\
Mid-level consult (99243) & 21.6 & 6 & 27 \\
Uterine fibroid embolization & 7.2 & 1.6 & \\
Iliac, femoral, or renal artery stent & 7.2 & 1.6 & \\
Hepatic artery chemoembolization & 7.2 & 1.6 & $\mathbf{\$}$ per Month \\
Projected Revenue & No. per Month & & $\$ 14,479$ \\
Uterine artery embolization & 7.2 & & $\$ 8,312$ \\
Iliac, femoral, or renal artery stent & 7.2 & & $\$ 10,936$ \\
Hepatic chemoembolization & 7.2 & & $\$ 2,456$ \\
Low-level follow-up (99212) & 81 & & $\$ 2,981$ \\
Mid-level consult (99243) & 27 & & $\$ 39,164$ \\
Total Average Revenue & &
\end{tabular}

*Assuming 30 minutes for a new patient, 15 minutes for an established patient, and a 3:1 ratio of follow-up patients to new patients, one will be able to schedule six new patients and 18 follow-up patients in an 8-hour day (not all may require to see a doctor). 
Table 3 Pro Forma

\begin{tabular}{|c|c|c|c|}
\hline \multirow[b]{2}{*}{ Costs } & \multicolumn{3}{|c|}{ Revenues } \\
\hline & Month 1 & Quarter 1 & Year 1 \\
\hline & $\$ 39,164$ & $\$ 117,492$ & $\$ 469,967$ \\
\hline Rent (1200 sq. ft. @ \$22.50/sq. ft.) & $\$ 2,250$ & $\$ 6,750$ & $\$ 27,000$ \\
\hline Amortized buildout, furniture, and supplies & $\$ 1,742$ & $\$ 5,225$ & $\$ 20,900$ \\
\hline Medical aid & $\$ 2,808$ & $\$ 8,424$ & $\$ 33,696$ \\
\hline Physician's assistant & $\$ 1,521$ & $\$ 4,562$ & $\$ 18,249$ \\
\hline Medical receptionist & $\$ 1,779$ & $\$ 5,336$ & $\$ 21,344$ \\
\hline Interventional radiologist & $\$ 15,513$ & $\$ 46,540$ & $\$ 186,160$ \\
\hline Computer hardware and information systems & $\$ 990$ & $\$ 2,970$ & $\$ 11,880$ \\
\hline Billing and collections & $\$ 1,175$ & $\$ 3,525$ & $\$ 14,099$ \\
\hline Profit/loss & $\$ 11,387$ & $\$ 34,160$ & $\$ 136,640$ \\
\hline
\end{tabular}

Assuming 30 minutes for a new patient, 15 minutes for an established patient, and a 3:1 ratio of follow-up patients to new patients, one will be able to schedule six new patients and 18 follow-up patients in an 8-hour day (not all may require to see a doctor).

${ }_{* *}^{*}$ Assumes $80 \%$ of new patients referred for one of the three services listed above will have indications for procedures and undergo procedure. *****Assumes standard reimbursement rates without excessive bad debt or uninsured population.

*** Does not consider revenue from other diagnostic imaging tests routinely ordered in the clinic, such as pelvic ultrasound, renal magnetic resonance imaging, computed tomography scans, or noninvasive vascular evaluations.

to contact consultants at any time, the telephone should be consistently answered and monitored 24 hours a day. In addition, it may be desirable to employ a medical assistant or nurse to obtain vital signs and organize charts and other clinical material that the physician will want to review. A PA or nurse practitioner (NP) can perform follow-up visits for routine, well patients. Although NPs can also accept referrals, using them for this vital role in maintaining the lifeline of the practice is usually not advisable.

A large interventional radiology practice can easily see patients 2 or 3 full days per week. Half that time might be taken up by doctors seeing new patients and doing short-term follow-up visits, with NPs or Pass providing long-term follow-up the remainder of the time. If a vein clinic is also done at the site, another 2 or more days may be required.

In general, physicians should be on site for at least a half day and can take turns staffing the clinic. If a practice is successful in promoting its doctors along service lines, then the clinic can be organized to accommodate those areas of interest. For example, a clinic meeting Tuesdays and Thursdays might schedule Tuesday as fibroid referral clinic, Thursday as peripheral arterial disease clinic, the following Tuesday as liver cancer clinic, and so on, with appropriate doctors scheduled for those days. This facilitates establishment of an identity within the referring community and promotes patient care.

\section{RISKS}

There are several risks if execution of the business strategy is not done carefully and thoughtfully. For example, if a practice takes the clinical concept to an extreme and is not selective in triaging referrals, they may find a low percentage of new patients are appropriate for therapeutic interventions. Therefore, financial results will be much less robust than those outlined using the assumptions above and may even be negative. If the physician extender is not instructed and trained on follow-up parameters for various services, the liability issue could be important. Similarly, if the physician extender is presented as the clinical face of the interventionalist radiology practice, especially for new patients, it is unlikely that the practice will be viewed as adding value to patient care. If promotion results in demand that exceeds the practice's capacity to handle, the practice may inadvertently generate more business for other practitioners. If work is sloppy, unreliable, or the clinic is irresponsible with follow-up, subsequent referrals may not materialize. If the practice does not promote itself tactfully, potential referral sources may become disenfranchised. Finally, if any of these steps results in an undesirable experience for referred patients or their physicians, there is the possibility that referrers will regret their decision to try something new and may simply stop referring.

\section{REFERENCE}

1. McGraw JK, Fricke B, Lippert JA, et al. Prospective evaluation of imaging and interventional procedures generated by an out patient interventional radiology office. J Vasc Interv Radiol 2004;15:S175 